\title{
Extraction and Antioxidant Activity of Polysaccharide from Salvia miltiorrhiza
}

\author{
Jia Guangtao* \\ School of Biology Science and Techology \\ Weifang Medical University \\ Weifang, China \\ Corresponding author, e-mail:guangtaojia@163.com
}

\author{
Zhao Hanqing \\ School of Science and Social Science, Shandong Transport \\ Vocational College \\ Weifang, China \\ e-mail:zhao-hanqing@hotmail.com
}

\begin{abstract}
Objective: Salvia miltiorrhiza is a traditional Chinese herb medicine and is widely used in clinic application. This study is intended to extract polysaccharides from Salvia miltiorrhiza by ultrasonic method in order to evaluate its antioxidation activity. 2. Methods: (1) Spectrophotometry is used to determine content of polysaccharides from Salvia miltiorrhiza; (2) Ultrasonic method was used to extract polysaccharides from Salvia miltiorrhiza and water as the solution. The effect of the different volume of solvent, extraction time and frequency were conducted (3) The antioxidation of Salvia miltiorrhiza polysaccharides is evaluated by pyrogallol autoxidation and deoxyribose degradation. 3. Results: (1) Phenol - sulfuric acid method was established to test the content of polysaccharide from Salvia miltiorrhiza. The results were in good linear relationship, and $Y=$ $0.9947 x+0.0451, R^{2}=0.9996$. (2) The extraction process of Salvia miltiorrhiza polysaccharides by ultrasonic method was 5 g raw material, $100 \mathrm{~mL}$ water solution, twice extraction, 30 min for each. (3) Antioxidtion tests indicated that with the concentration $0.05 \mathrm{mg} / \mathrm{mL}$ of polysaccharides, the inhibition rate for superoxide anion radical could reach $1.28 \%$ and $13.54 \%$ for hydroxyl radical, respectively. And the reducing power was likely to increase with the increasing of polysaccharides concentration.

4. Conclusion: Ultrasonic method could effectively extract polysaccharide from Salvia miltiorrhiza. Antioxidation tests indicate that polysaccharides from Salvia miltiorrhiza have a certain removal effect on superoxide anion radical and hydroxyl
\end{abstract} radical.

Keywords: Salvia miltiorrhiza; polysaccharides; Ultrasonic extraction; Phenol-sulfuric acid method; antioxidation activity

\section{INTRODUCTION}

Salvia miltiorrhiza is a traditional Chinese herb medicine. It is mainly used in treatment of stroke, cardio-vascular disease, hyperlipidemia, and cerebro-vascular disease, etc ${ }^{[1-3]}$. At present, studies toward Salvia miltiorrhiza activity are mainly concentrated on salvianolic acid, tanshinones and caffeic acid and their pharmacology function ${ }^{[4]}$. Polysaccharides compound is a significant bioactive substance which can enhance immune function for antitumor, anticoagulation, blood fat regulation, anti-aging, anti-virus, etc ${ }^{[5,6]}$. The biological function of polysaccharides is closely related to cleaning power of active oxygen radicals. Therefore, polysaccharides gained a large scale of popularity. However, fewer studies are on polysaccharides from Salvia miltiorrhiza which is $12 \% \sim 38 \%$ in it ${ }^{[7]}$.
This study is intended to extract polysaccharides from Salvia miltiorrhiza by ultrasonic method and evaluate their antioxiditon activity, in order to provide reliable data and information for further development of Salvia miltiorrhiza resources.

\section{MATERIALS AND METHODS}

\section{A. Maiterials}

\section{1)Herb Medicine}

Salvia miltiorrhiza purchased from Beijing Tong Ren Tang Group Co., Ltd., was identified by Chinese medicine teaching and research section of Weifang Medical university.

2) Reagents

Ethanol(95\%), hydrochloric acid, sulfuric acid, glucose, $\mathrm{FeSO}_{4}$-EDTA $(10 \mathrm{mmol} / \mathrm{L}), 2$ 2-deoxyribose $(10 \mathrm{mmol} / \mathrm{L})$, phosphate buffer $(\mathrm{pH}=7.4,0.1 \mathrm{~mol} / \mathrm{L}), \mathrm{H}_{2} \mathrm{O}_{2}$ $(10 \mathrm{mmol} / \mathrm{L})$, TCA $(2.8 \%)$, TBA $(1.0 \%)$, phenol $(6 \%$ and $80 \%$ ), distilled water, chloroform, n-butyl alcohol, Tris-HCl buffer $(\mathrm{pH}=8.2)$, pyrogallic acid $(45 \mathrm{mmol} / \mathrm{L}$ pyrogallol soluted in $10 \mathrm{mmol} / \mathrm{L}$ HC1), vitamin C $(0.024$ $\mathrm{mg} / \mathrm{mL}$ ), all the regents used in this study were analytical pure.

\section{B. Methods}

1)Phenol-sulfuric acid method for determining polysaccharide content

a) Extraction of polysaccharide : Salvia miltiorrhiza powders mixed with distilled water in proportion by ultrasonic extraction. Then centrifugal separation method was used to obtain supernatant. The residue was treated by the flow of the above process and the supernatants were mixed. Seveage method was used to get rid of protein after the mixed supernatants were concentrated. The process of alcohol precipitation, centrifugal separation, residue drying was performed to gain crude polysaccharide from Salvia miltiorrhiza.

b) Standard curve for polysaccharide: Glucose $(0.0050 \mathrm{~g})$ was dissolved into distilled water $(50 \mathrm{~mL})$ to gain standard glucose solution. Different volumes of standard glucose solution $(0.1,0.2,0.3,0.4,0.5$ and $0.6 \mathrm{~mL}$ ) were put into 6 tubes, and distilled water was added to $2.0 \mathrm{~mL}$. Then phenol $(6 \%, 1 \mathrm{~mL})$ and $5 \mathrm{~mL}$ sulfuric acid were added into each tube by order, and standing in room temperature for $25 \mathrm{~min}$. The solutions were tested with an ultraviolet spectrophotometer (Shanghai Meipuda Instrument Co., LTD) at 490nm. 
Distilled water $(1 \mathrm{~mL})$ as the blank control solution was prepared with the method mentioned above. Taking the absorbance $\mathrm{A}$ as the longitudinal coordinate and the glucose concentration $\mathrm{C}$ as the horizontal coordinate, the regression equation was obtained, and the standard curve was established.

c) Determination of polysaccharide content from Salvia miltiorrhiza: Crude drying polysaccharide products $0.05 \mathrm{~g}$ dissolved into distilled water $500 \mathrm{~mL}$ to obtain the sample solution, and then treated with the method mentioned in B. a) The absorbance was recorded with ultraviolet spectrophotometer to calculate the content of polysaccharide :

$\mathrm{Y}(\%)=\mathrm{M} / \mathrm{W} \times 100 \%$

Where $\mathrm{Y}$ is the yield ratio of polysaccharide (\%); $\mathrm{M}$ is the measured quantity of crude polysaccharide (g); $\mathrm{W}$ is original weigh of Salvia miltiorrhiza powders.

\section{2)Ultrasonic extraction of polysaccharide}

Effect of different volumes of solvent, extraction time and extraction frequency was test by ultrasonic extraction. Crude polysaccharides were prepared by the method mentioned yin B. a). The yield ratio of polysaccharide with three factors was calculated, respectively

3) Determination of in vitro antioxidant capacity of Salvia miltiorrhiza polysaccharides

a)Determination of superoxide anion radical scavenging capacity: $9 \mathrm{~mL}$ Tris- $\mathrm{HCl}$ buffer $(\mathrm{pH}=8.2)$ was added into a tube, insulation for $20 \mathrm{~min}$ in a water bath thermal; then $0.5 \mathrm{~mL}$ preheated $\left(25^{\circ} \mathrm{C}\right)$ pyrogallic acid was mixed with the solution above, and poured into colorimetric tube immediately. After 3min, vitamin C $(0.5 \mathrm{~mL})$ was added into the colorimetric tube and kept for $5 \mathrm{~min}$ in room temperature. The absorbance $\mathrm{D}_{0}$ was recorded at $420 \mathrm{~nm}$, which was the autoxidation rate of pyrogallic acid. And the final volume of the reaction system was $10 \mathrm{~mL}$.

Sample solution of crude polysaccharide was added into Tris-HCl buffer solution, and the sample process mentioned above was performed, then the absorbance $\mathrm{D}_{1}$ was recorded at $420 \mathrm{~nm}$. Sample solution of crude polysaccharide was replaced by distilled water to be the blank control $\left(D_{2}\right)$. The following equation was to quantify the inhibition rate of superoxide anion radical:

Inhibition rate $(\%)=\frac{D_{0}-\left(D_{1}-D_{2}\right)}{D_{0} \times 100} \times 100 \%$

b) Determination of hydroxyl radical scavenging capacity: $\mathrm{FeSO}_{4}$-EDTA mixture $(0.2 \mathrm{~mL})$ and 2-deoxyribose $(0.5 \mathrm{~mL})$ were mixed into a tube; then different concentration of sample solution of crude polysaccharide $(0.5 \mathrm{~mL})$ was added into the tube; $\mathrm{H}_{2} \mathrm{O}_{2}$ $(0.2 \mathrm{~mL})$ was put into the tube after using phosphate buffer to get the volume to $1.8 \mathrm{~mL}$; and the tube insulation for 20min in a water bath thermal. Furthermore, TCA $(1.0 \mathrm{~mL})$ and TBA $(1.0 \mathrm{~mL})$ were mixed and put into the tube, then placed into boiling water bath for $15 \mathrm{~min}$. At last, the absorbance $\left(\mathrm{A}_{2}\right)$ at $532 \mathrm{~nm}$ was recorded after the tube was cool down. Sample solution of crude polysaccharide was replaced by distilled water to be the blank control $\left(\mathrm{A}_{1}\right)$. The following equation was to quantify the inhibition rate of superoxide anion radical:
Inhibition rate $(\%)=\left(A_{1}-A_{2}\right) / A_{1} \times 100 \%$

\section{RESULTS}

A. Establishment of quantitative standard curve of Salvia miltiorrhiza polysaccharides

With different concentration of standard glucose solution added with phenol-sulfuric acid, absorbance was determined at 490nm for establishment of quantitative standard curve of Salvia miltiorrhiza (Fig. 1). With fitting, calculation formula of Salvia miltiorrhiza polysaccharides was as follow $y=0.9947 x+0.0451$. A good linear relationship was shown in Fig 1 , with $\mathrm{R}^{2}=0.9996$, which can satisfy the needs for determination of Salvia miltiorrhiza polysaccharides.

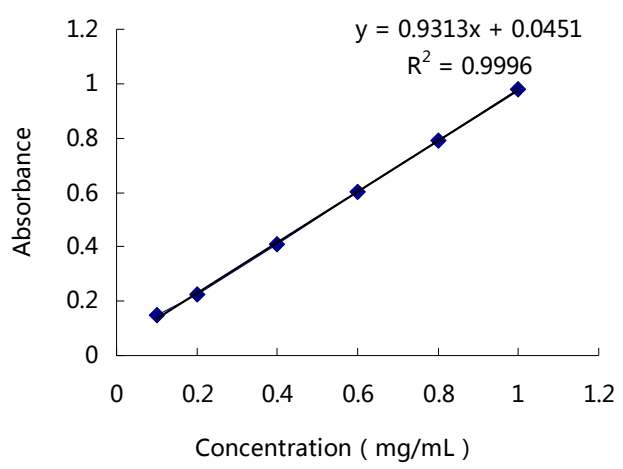

Figure 1. Quantitative standard curve of Salvia miltiorrhiza polysaccharides.

B. Process of Salvia Miltiorrhize Polysaccharides Extraction by Ultrasonic Waves

1) Effect on polysaccharides extraction yield by solution volume: With fixed amount of Salvia miltiorrhiza and different quantity of water solution for ultrasonic extraction, the results are shown in Fig. 2. When solution amount was $100 \mathrm{~mL}$, extraction rate was the best. With an increasing amount of solution appliance, extraction rate was slightly rather than obviously decreasing. Therefore, the best solution amount is $100 \mathrm{~mL}$ with $5 \mathrm{~g}$ Salvia miltiorrhiza.

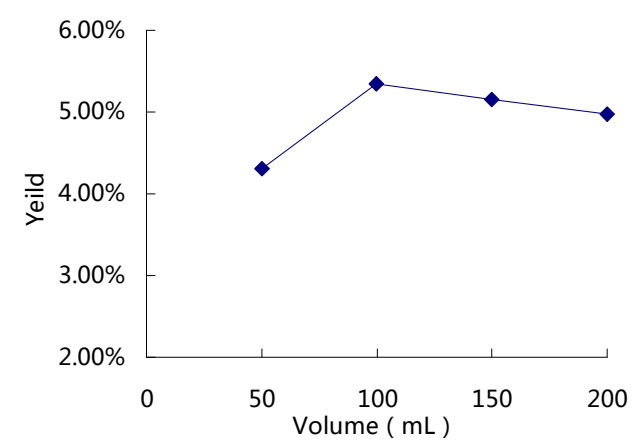

Figure 2. Effect on extraction quantity by solution volume

2) Effect on polysaccharides extraction yield by extraction time

Effect on polysaccharides extraction ratio in different time duration, see Fig. 3. The results indicate that extraction ratio is obviously increased with time goes by. 


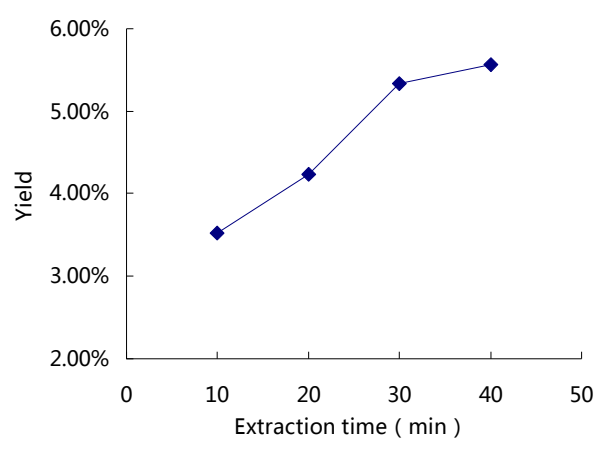

Figure 3. Effect on polysaccharides extraction yield by extraction time

3) Effect on polysaccharides extraction yield by extraction frequency

Effects are shown in Fig. 4 about frequent extraction in solutions with one portion of Salvia miltiorrhiza powder. The results indicate that with an increasing frequency of extraction, the ratio was likely to increase. For the second time extraction, the ratio was 5.84\%, while the data was $5.97 \%$ in the fourth time extraction. With a slight difference in between, and for economical purpose, two times extraction was recognized as the best frequency of extraction.

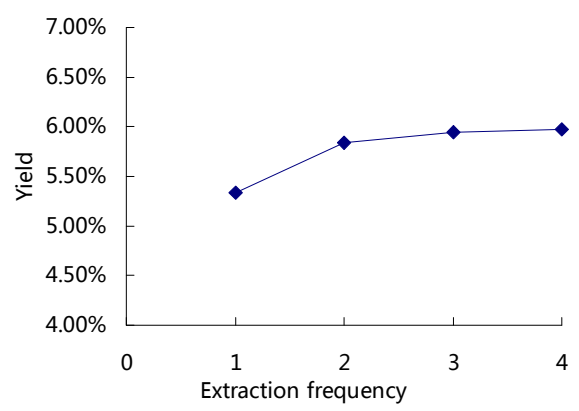

Figure 4. Effect on polysaccharides yield by extraction frequency

\section{Results for Polysaccharides Antioxidant Activity Determination}

1) Ability to remove superoxide anion

Different samples, with reagents added in, were determined by spectrophotometer for absorbance. Three tests were applied on each sample before calculating their cleaning power for superoxide anion radicals by the polysaccharides under different concentrations based on formulate (2). The results can be seen in Fig. 5. The results indicated that polysaccharides of Salvia miltiorrhiza had certain cleaning power to superoxide anion radicals. When the concentration of polysaccharides solution was $0.01 \mathrm{mg} / \mathrm{mL}$, the inhibition rate was $1.19 \%$. When the concentration was $0.05 \mathrm{mg} / \mathrm{mL}$, the rate was $1.28 \%$. In addition, the inhibition rate was increasing constantly with the increasing of polysaccharides concentration within the above range.

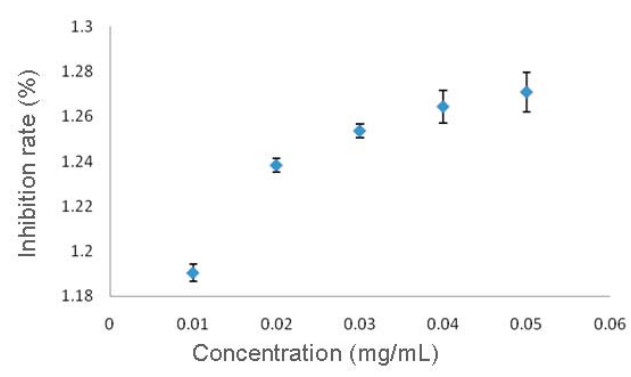

Figure 5. Ability of Polysaccharides to remove superoxide anion radicals

\section{2) Ability to remove hydroxyl radicals}

Different samples, with reagents added in, were determined by spectrophotometer for absorbance. Three tests were applied on each sample before calculating their cleaning power for hydroxyl radicals by the polysaccharides under different concentrations based on formulate (3). The results can be seen in Fig. 6. The results indicated that polysaccharides of Salvia miltiorrhiza had certain cleaning power to hydroxyl radicals. When the concentration of polysaccharides solution was $0.01 \mathrm{mg} / \mathrm{mL}$, the inhibition rate was $6.48 \%$. When the concentration was $0.05 \mathrm{mg} / \mathrm{mL}$, the rate was $13.54 \%$. In addition, the inhibition rate was increasing constantly with the increasing of polysaccharides concentration within the above range.

\section{CONCLUSION}

This research adopted ultrasonic extraction for polysaccharides in Salvia miltiorrhiza. The crude polysaccharides were obtained by centrifugation, concentration, and alcohol precipitation, etc. Glucose was taken as the reference for the establishment of quantitative standard curve of Salvia miltiorrhiza polysaccharides by adopting phenol-sulfuric acid

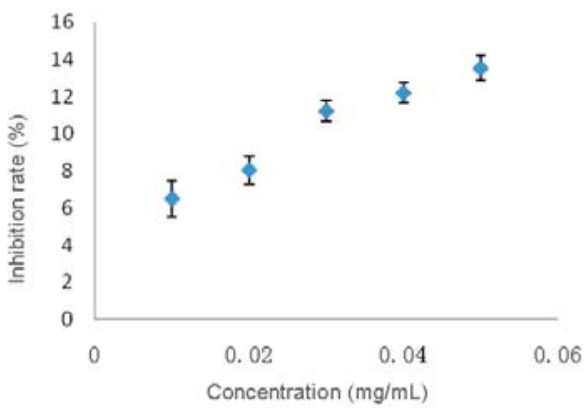

Figure 6. Ability of Polysaccharides to remove hydroxyl radicals

colorimetry. The results were in good linear relationship, and can meet the quantitative requirements for crude polysaccharides.

When ultrasonic was used for polysaccharides extraction, the extracting ratio slightly decreased while the solution was increased. Since the extraction ratio did not change much, this result was possibly because of the increased solution quantity and processing amount. The best option was 1g Salvia miltiorrhiza powder for $100 \mathrm{~mL}$ solution. The extraction ratio was promoted prominently for the first and second extraction, while the ratio was increasing slowly to a fixed number for the third and fourth extraction. From the economical and practical 
aspects, two times of extraction was taken as the most ideal frequency.

To certain extent, Salvia miltiorrhiza polysaccharides were useful to clean superoxide anion radicals and hydroxyl radicals. The cleaning power was increased with the increased concentration of polysaccharides.

\section{ACKNOWLEDGEMENTS}

Supported by a project grant from the Foundation for Outstanding Young Scientist in Shandong Province (Grand No. BS2013YY063).

\section{REFERENCE}

[1] X. C. Ge and J. Y. Wu. Tanshinone production and isoprenoid pathways in Salvia miltiorrhiza hairy roots induced by $\mathrm{Ag}^{+}$and yeast elicitor. Plant Science, 2005, 168: 487-491.
[2] H. Wang, Q. Wang, H. M. Luo, et al. Quantitative analysis of Salvia miltiorrhiza polysaccharide and determination of its composition by HPCE. Chinese Archives of Traditional Chinese Medicine, 2007, 25(4): 827-829.

[3] T. Cheng. Cardiovascular effects of Danshen. International Journal of Cardiology, 2007, 121(4): 9-22.

[4] R. W. Jiang, K. M. Lau, P. M. Hon, et al. Chemistry and biological activities of caffeic acid derivatives from Salvia miltiorrhiza. Current Medical Chemistry, 2005, 12(2): 237-246.

[5] M. Y. Xie and S. P. Nie. A review about the research of structure and function of polysaccharides from natural products. Journal of Chinese Institute of Food Science and Technology, 2010, 10(2): $1-10$.

[6] L. H. Shen, J. S. Wang, Y. Li, et al. Research and application of plant polysaccharide. Chinese Agricultural Science Bulletin, 2011, 27(02): 349-352.

[7] X. Y. Wei, M. B. Jing, J. C. Wang, et al. Preliminary study on Salvia miltiorrhiza bung endophytic fungus. Academic Journal of Xi'an Jiaotong University, 2010, 22(4): 241-246. 